EPJ Web of Conferences 47, 03007 (2013)

DOI: $10.1051 /$ epjconf/20134703007

(C) Owned by the authors, published by EDP Sciences, 2013

\title{
Searching for planetary transits around $M$ dwarfs with telescope networks
}

\author{
Enric Palle $e^{1,2, a}$, Victor J.S. Béjar ${ }^{1,2, b}$ and Estefanía Casal ${ }^{2, c}$ \\ ${ }^{1}$ Instituto de Astrofisica de Canarias (IAC), via Lactea s/n 38200, La Laguna, Spain \\ ${ }^{2}$ Departamento de Astrofisica, Universidad de La Laguna, Spain
}

\begin{abstract}
As of today, hundreds of extrasolar planets have been discoverd, and thousands of candidates are waiting for confirmation. Although we already have discovered dozens of the least massive planets (the so called super-Earths), only a few have been found to be orbiting low mass stars, and only one that actually transist a bright $\mathrm{M}$ star. Here, we propose to carry out a photometric search for transiting super-Earths in the Habitable Zone of a large sample of very low-mass stars $\left(\mathrm{M}<0.3 M_{\odot}\right)$. These planets have a higher transit detection probability, shorter orbital periods and produce larger radial velocity amplitudes than those orbiting Solar type stars. The observing strategy proposed for such a search would require a robotic network of 1-m class telescopes, such as the LCOGT will provide.
\end{abstract}

\section{INTRODUCTION}

Since the discoveries of the first extrasolar planets around the pulsar PSR $1257+12$ by the timing method (Wolszczan \& Frail 1992) and around the Solar type star 51 Peg by radial velocity (Mayor \& Queloz 1995) more than 800 planets have been confirmed. Most of them have been identified using the successful radial velocity technique, but during last years an increasing number of extrasolar planets have been discovered transiting their host stars (Konacki et al. 2003) and by direct imaging (Chauvin et al. 2004). Most specially since the implementation of the Kepler mission. In particular, since the discovery of the first transiting planet (Charbonneau et al. 2000), this technique has provided precise measurements of the radii and masses of extrasolar planets and the detection of direct emission light from the planetary secondary eclipses in a few cases (Deming et al. 2005; Charbonneau et al. 2005). These observations have led to the identification of molecular species in their atmospheres like $\mathrm{H}_{2} \mathrm{O}$ or $\mathrm{CH}_{4}$ (Tinetti et al. 2007; Swain et al. 2008) similar to those found in the atmospheres of our Solar System planets and free-floating brown dwarfs.

Recently, planets even less massive than $10 M_{\text {Earth }}$ (so called super-Earths) have been discovered around main sequence stars (Rivera et al. 2005), and the first transiting super-Earths: COROT-7b (Leger et al. 2009) and GJ1214b (Charbonneau et al. 2009) have been found. The statistics from the recent Kepler database also point to a large population of rocky planets around M stars (Dressing \& Charbonneau 2013).

Although many efforts in extrasolar planet searches have been done during last decade, very few planets are known around very low-mass stars. Radial velocities studies using HARPS and transit investigations from the Kepler satellite have led to the preliminary conclusion that super-Earths around

\footnotetext{
ae-mail: epalle@iac.esr

be-mail: vbejar@iac.es

ce-mail: estefania.casal@yahoo.es
}

This is an Open Access article distributed under the terms of the Creative Commons Attribution License 2.0, which permits unrestricted use, distribution, and reproduction in any medium, provided the original work is properly cited. 
early M dwarfs are significantly more frequent than giant planets and tend to appear in multiplanetary systems (Bonfils et al. 2007; Dressing \& Charbonneau, 2013). The discovery of a Earth-like planet in the Habitable Zone (HZ), defined as the region where liquid water can exists, is currently one of the major goals in Astrophysics and Science in general.

\section{THE LAS CUMBRES OBSERVATORY GLOBAL NETWORK}

Las Cumbres Observatory Global Telescope Network (LCOGT) is a nonprofit corporation, which is developing a global network of observing facilities at premier astronomical sites. The global network will consist of between 12 and 18 robotic telescopes with $1 \mathrm{~m}$ aperture, and between 16 and 22 robotic telescopes with $40 \mathrm{~cm}$ aperture. The scientific aims of LCOGT lie within time-domain astronomy. This encompasses anything that moves or that changes its brightness or spectral energy distribution with time. LCOGT specializes this definition to mean that the observatory's main interests include observation and interpretation of (a) Extrasolar planets, including observations of transits, microlensing events, and radial velocities, (b) Supernovae and gamma-ray bursts (GRBs) of all types, including both photometric and spectrographic studies, and (c) Variable stars, including pulsating stars and binary and multiple star systems.

Such a telescope netwrok, with observing nodes at both the Northern and Southern hemisphers could provide continuos observations of an object from days to months.

\section{A TARGETED SEARCH FOR ROCKY PLANETS AROUND THE BRIGHTES M STARS WITH LCOGT}

By using LCOGT, we propose to carry out a photometric search for transiting super-Earths around very low-mass stars $\left(\mathrm{M}<0.3 M_{\odot}\right)$. This roughly correspond to spectral types M4 and later. Given the small radii $\left(\mathrm{R}<0.3 M_{\odot}\right)$ of these objects, the depth of the transit, which depend on the ratio of the planet and star radius $\left(\Delta F \sim R_{p} / R_{*}\right)$ is higher than in Solar type stars, and hence, easier to detect. For example a transiting Earth-like planet will produce a decrement of 0.08 mmag in the flux of a star like the Sun, while this is larger than $1 \mathrm{mmag}$ for intermediate and late $\mathrm{M}$ stars.

The probability of finding a transit in the $\mathrm{HZ}$ is also much higher in very low-mass stars than around solar type stars. This quantity $\left(P_{\text {tran }}\right)$ can be approximated by $P_{\text {tran }} \sim a / R_{*}$, where a is the orbital separation, which at the $\mathrm{HZ}$ is $a \sim \sqrt{L u m} \sim R_{*} T_{\text {eff }}^{2}$, and hence $P_{\text {tran }} \sim T_{\text {eff }}^{-2}$. For example a transiting Earth-like planet has a probability of only $0.5 \%$ to be detected in the HZ of a solar type star, while this is larger than $2 \%$ for intermediate and late $\mathrm{M}$ stars. Another advantage is that the amplitude of the radial velocity variations induced by the planet around very low-mass stars is larger and hence, it is easier to confirm them using this method. For example an Earth-like planet in the HZ produce a radial velocity variation of $10 \mathrm{~cm} / \mathrm{s}$, while this is larger than $1 \mathrm{~m} / \mathrm{s}$ in intermediate and late $\mathrm{M}$ stars. These transiting super-Earths around very low-mass stars will be located at separations lower than $0.05 \mathrm{AU}$ and have orbital periods lower than 10 days. Their transit duration is estimated to be below 3 hours, assuming a circular orbit.

Casal et al. (in prep.) have produced a list of nearby $M$ dwarf from a compilation and crosscorrelation of the PMSU, RECONS, UltraCool Dwars and Lepine and Gaidos catalogues. For each star, mass, radius, luminosity and $T_{\text {eff }}$ were estimated. Based on this information and using probability distributions, Casal et al estimate that there is a $85 \%$ probability of finding one transiting superearth in the $\mathrm{HZ}$ of the nearest $\sim 350 \mathrm{M}$ stars, which are located at a distance lower than $\sim 11 \mathrm{pc}$. Very similar numbers are derived by Dressing \& Charbonneau (2013) based on statistical analisis of Kepler exoplanet candidates.

In order to carry out our search for transiting super-Earth, we plan to perform a long-term monitoring program of this sample, using differential photometric techniques. Initial test runs with GJ $896 \mathrm{~A}$ and GJ896 B (Béjar, priv. comm.) indicate that one can obtain precisions below 1 mmag in time scales of a 
few minutes in 1-meter class telescopes. A search in astronomical databases indicates that $98 \%$ of our sample of $\mathrm{M}$ dwarfs have at least one bright $(\mathrm{J}<13)$ reference star and $80 \%$ of them have more than one in an $8^{\prime} \times 8^{\prime}$ area.

Nearby bright cool stars are evenyl distributed on the sky. Thus, the proposed observing strategy would be to continuosly phptometrically monitor a given star for a period of 15 days (360 hours). About 4 stars could be monitored simultanously by visiting each star during 15 minutes per hour. At this rate, 27,000 observing hours would be needed to complete such a program. For a single telescope, considering no time losses due to weather or aliases between the Earth and the star's rotational periods, nine years woulb be needed to complete such a program. On the contrary, for a telescope network such as LCOGT (supposing 15 geographycally-distributed 1-m telescopes), it would take $60 \%$ of the total network observing time over one year only. Nevertheless, the observations would probably need to be scheduled over a period of 2 to 3 years.

\section{References}

[1] Bonfils, X. et al., A\&A, 474, 293 (2007)

[2] Charbonneau, D. et al., ApJ, 529, L45 (2000)

[3] Charbonneau, D. et al., ApJ, 626, 523 (2005)

[4] Charbonneau, D. et al., Nature, 462, 891 (2009)

[5] Chauvin G. et al., A\&A, 425, L29 (2004)

[6] Deming, D. et al., Nature, 434, 740 (2005)

[7] Dressing, D. \& Charbonneau, D., ApJ (in press) (2013)

[8] Konacki et al., Nature, 421, 507 (2003)

[9] Leger, A. et al., A\&A (2009)

[10] Mayor \& Queloz, Nature, 378, 355 (1995)

[11] Queloz, D. et al., A\&A (2009)

[12] Rivera, E. et al., ApJ, 634, 625 (2005)

[13] Swain, M. et al., Nature, 452, 329 (2008)

[14] Tinetti, G., et al., Nature, 448, 169 (2007)

[15] Wolszczan \& Frail, Nature, 355 (1992) 